\title{
Small-amplitude hunting diagnosis method for high-speed trains based on the bogie frame's lateral-longitudinal-vertical data fusion, independent mode function reconstruction and linear local tangent space alignment
}

Proc IMechE Part F:

J Rail and Rapid Transit

2019, Vol. 233(10) 1050-1067

(C) IMechE 2019

Article reuse guidelines:

sagepub.com/journals-permissions DOI: 10.1 I77/09544097/88254I2

journals.sagepub.com/home/pif

@SAGE

\author{
Yunguang $Y e^{1,2}$ (D) and Jing Ning'
}

\begin{abstract}
Hunting stability is an important factor that influences the running safety of high-speed trains. Most of the existing hunting monitoring methods monitor only the standard hunting. The small-amplitude hunting, however, not only affects ride comfort but also aggravates the wheel-rail wear. Therefore, to extend the service life of wheels and rails and to improve the ride comfort, it is extremely important to monitor the small-amplitude hunting. Hunting motion is a coupled movement of lateral and yaw displacements of the wheelset. When the bogie is in an unstable state, instability will occur not only in the lateral side but also in the longitudinal and vertical sides of the bogie. To improve the robustness of the small-amplitude hunting monitoring methods, this study proposes an idea of the bogie frame's lateral-longitudinalvertical data fusion. In addition, the small-amplitude hunting signals have strong nonlinear characteristics, and their frequency and amplitude are unstable. Using only the amplitude or frequency to detect the small-amplitude hunting has obvious shortcomings. Therefore, a new feature extraction method based on the independent mode function reconstruction and linear local tangent space alignment (IMFR-LLTSA) is proposed. This method has been tested with three simulated signals. Finally, a method of combining the bogie frame's lateral-longitudinal-vertical data fusion and IMFR-LLTSA is used to identify the small-amplitude hunting of high-speed trains. This method has been validated using the data of the CRH380a high-speed train running on the Shanghai-Hangzhou line, monitored by the authors' research group. The results show that this method is superior to the single lateral diagnosis method.
\end{abstract}

\section{Keywords}

Small-amplitude hunting, lateral, longitudinal, vertical, independent mode functions reconstruction, linear local tangent space alignment

Date received: 10 July 2018; accepted: 29 December 2018

\section{Introduction}

Hunting motion and small-amplitude hunting motion

The conical profile of railway wheelsets is a great invention in the field of railways, which helps trains pass a curved track smoothly by changing the rolling radii of the wheels. The existence of the wheelset taper, however, also results in a serious shortcoming. When a vehicle runs on a straight track at high speed, and the wheel is subjected to external stimuli, the tapered wheelset will shift and cause the rolling radii of its left and right wheels to change. The changes in the rolling radii will lead to unequal line speeds of the left and right wheels, and further result in a kinematic oscillation of the wheelset ${ }^{1,2}$ (see Figure 1(a)). This vibration is superposed on the steady rolling of the wheelset and represents a $3 \mathrm{D}$ motion, which is described as a coupled movement of lateral and yaw displacements of the locomotive wheelset relative to the central line of the track that it runs on

\footnotetext{
'School of Mechanical Engineering, Southwest Jiaotong University, Chengdu, China

${ }^{2}$ Institute of Land and Sea Transport Systems, Technical University of Berlin, Berlin, Germany

Corresponding author: Jing Ning, Southwest Jiaotong University, Chengdu 61003I, Sichuan, China.

Email: ningjing@swjtu.edu.cn
} 


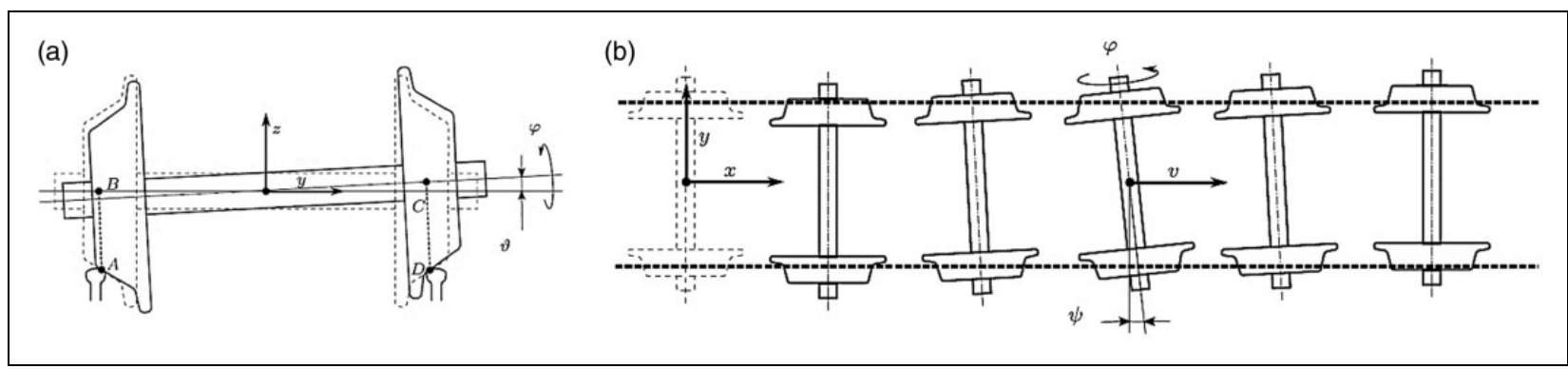

Figure I. Hunting motion. ${ }^{3}$ (a) Change of the rolling radii on a curved track; (b) Kinematic oscillation on a straight track.

(see Figure 1(b)), namely hunting motion. ${ }^{3,4}$ During the actual running of vehicles, hunting motion will decrease the ride quality of passengers and increase the dynamic loads imposed on every part of vehicles. ${ }^{5}$ In addition, severe hunting motion will cause violent impacts on tracks and even may result in derailments of vehicles, thereby threatening the running safety. ${ }^{6-8}$

To prevent a vehicle from hunting instability, the running speed of the vehicle needs to be controlled below the critical speed. However, the vehicle system has a lot of nonlinear factors including characteristics of yaw dampers and suspensions, and geometric contact characteristics of wheels and rails. ${ }^{9-16}$ Furthermore, the actual running conditions of vehicles vary greatly. ${ }^{17}$ These nonlinear factors and complex running conditions mean that when a vehicle runs below the theoretical critical speed, the vehicle is also prone to a smallamplitude oscillation. ${ }^{18-20}$ In this paper, we call this phenomenon as small-amplitude hunting.

With regard to the issues concerning small-amplitude hunting, D'Souza and Tsung ${ }^{21}$ researched the hunting motion of a full vehicle through the function description method and proposed that during the early stage of the hunting motion, a small-amplitude limit-cycle oscillation occurred on the wheelset. Starting from the wheel-rail contact characteristics, by researching the bifurcation characteristics of hunting, Polach ${ }^{18,19}$ indicated that under the condition of supercritical bifurcation, a small limit-cycle oscillation would occur when the vehicle speed is below the critical speed of hunting instability. Through theoretical derivation and analysis of massive measured data, $\mathrm{Cai}^{22}$ indicated that when the peak value of the bogie frame's lateral acceleration signal did not reach $8-10 \mathrm{~m} / \mathrm{s}^{2}$ for six consecutive times, the signal was seriously unstable or exceeded $2 \mathrm{~m} / \mathrm{s}^{2}$ many times. In actual running, the vehicle could also be thought at the hunting state. In addition, when researching measured signals of trains, the authors' research team ${ }^{23,24}$ found that when the CRH 380a train was running at $330-350 \mathrm{~km} / \mathrm{h}$, and the speed did not increase significantly, the motion with amplitude smaller than the standard limit-cycle amplitude of the hunting instability often occurred in the bogie frame's lateral vibration signal. Besides, the authors found that when a vehicle was in the small-amplitude hunting state, the peak value of a part of the bogie frame's lateral signal, sometimes, was less than $2 \mathrm{~m} / \mathrm{s}^{2}$.

The definition of small-amplitude hunting proposed in this paper is that the peak value of the bogie frame's lateral acceleration does not reach (or exceed) $8 \mathrm{~m} / \mathrm{s}^{2}$ for six consecutive times, but exceeds $2 \mathrm{~m} / \mathrm{s}^{2}$ many times and is seriously unstable (see Figure 2). Small-amplitude hunting not only affects the ride quality of passengers but also aggravates the wheel-rail wear. In addition, it is also a sign of standard hunting. It may evolve into standard hunting instability over time, greatly affecting the running safety of vehicles. ${ }^{25}$ The monitoring of the abnormal condition of the small-amplitude hunting can not only extend the service life of vehicles and infrastructures but also provide a precaution method of intensive hunting (standard hunting).

\section{Small-amplitude hunting monitoring method and problem statement}

In order to ensure the safe operation of vehicles, UIC518-2005, ${ }^{26}$ TSI L84/132-2008, ${ }^{27}$ UIC515, ${ }^{28}$ EN14363, ${ }^{29}$ etc. have stipulated that the parameters such as the peak value of bogie's lateral acceleration and wheel-rail lateral force must be less than the specified limit values. TB/T $3188-2007^{30}$ has stated that when the peak value of the bogie frame's lateral acceleration reaches or exceeds the limit value $8-10 \mathrm{~m} / \mathrm{s}^{2}$ (applicable to the design of the bogies) for consecutive six times, the vehicle can be determined to be in the hunting instability state. In the above standards, only the criterion of standard hunting is specified, but the small-amplitude hunting is not involved.

However, as the speed of trains increases, the small-amplitude hunting begins to occur frequently. In recent years, this phenomenon is especially evident in China, because its passenger cars' speed often exceeds $300 \mathrm{~km} / \mathrm{h} .{ }^{31}$ To monitor the smallamplitude hunting, Sun ${ }^{25}$ used the energy method to identify the small-amplitude hunting state and proposed a modification suggestion of the hunting motion evaluation method. Overall, at present, most existing studies only achieved the monitoring of the standard hunting, but they study little about the small-amplitude hunting. 


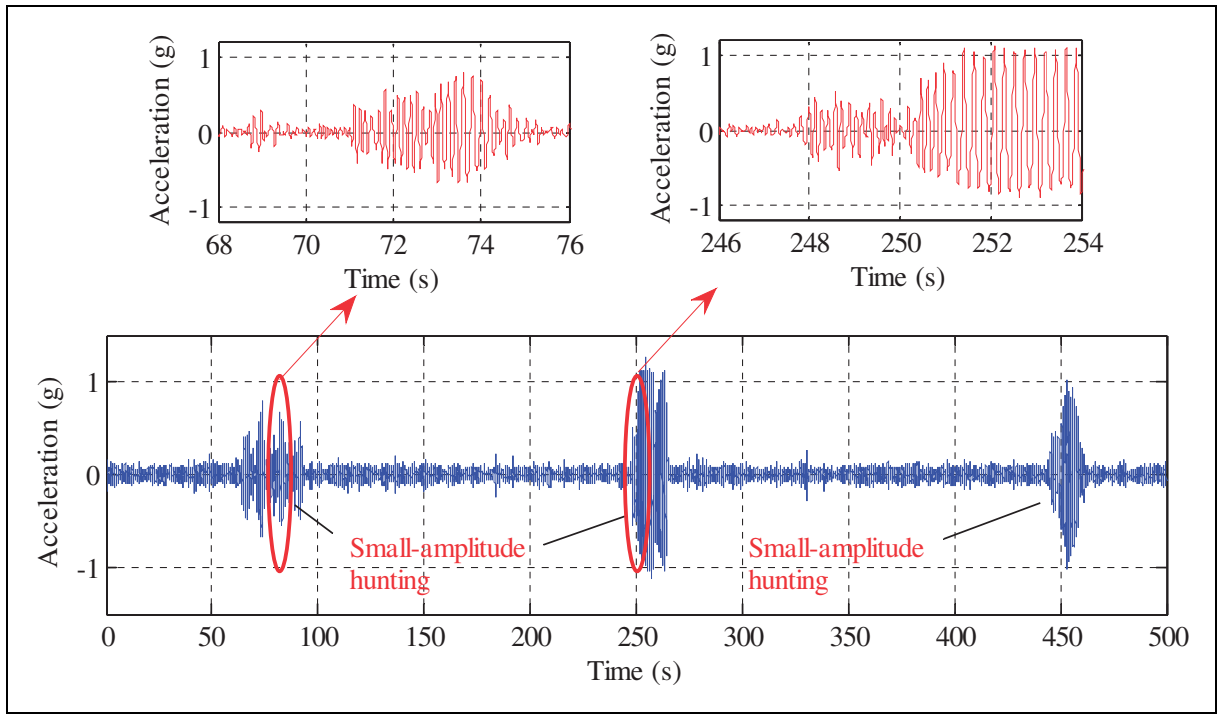

Figure 2. Lateral acceleration signal of the bogie frame when the small-amplitude hunting motion occurs during a test.

Besides, from the kinematic analysis (see Figure 1), it can be known that the hunting motion is a $3 \mathrm{D}$ coupled movement of lateral displacement and yaw of the wheelset around the central line of the track. When a vehicle is in a hunting state, the unstable phenomenon will occur not only in the lateral sides of bogies but also in the longitudinal and vertical sides. ${ }^{3,5,23}$ When processing the train's experimental data and analyzing its time-frequency features through Hilbert-Huang transformation (HHT), Ye et al. ${ }^{32}$ found that when the train is in small-amplitude and standard hunting states, the longitudinal and vertical vibration signals also contain instability information.

Through the literature review, it can be known that the current studies concerning the hunting monitoring method have two shortcomings as follows:

1. With the increase of railway vehicles' speed, especially for passenger cars, the phenomenon of small-amplitude hunting has begun to appear frequently. However, the monitoring of this phenomenon has not been mentioned in existing standards.

2. Hunting motion is a $3 \mathrm{D}$ coupled movement of lateral displacement and yaw of the wheelset. When the hunting motion occurs, the unstable phenomenon is shown not only in the lateral vibration signal but also in the longitudinal and vertical signals. The existing hunting monitoring methods only use the lateral signal to judge the hunting states, without making use of the longitudinal and vertical vibration signals. However, when a vehicle is in the small-amplitude hunting state, the peak value of a part of the bogie frame's lateral signal, sometimes, is less than $2 \mathrm{~m} / \mathrm{s}^{2} .{ }^{22}$ Therefore, using the single lateral-peak-value monitoring method to judge normal and smallamplitude hunting states cannot achieve an ideal result.
To monitor the small-amplitude hunting, an idea of integrating the bogie frame's lateral, longitudinal, and vertical signals is proposed in this paper, namely the bogie frame's lateral-longitudinal-vertical data fusion.

\section{Feature extraction method}

Since small-amplitude hunting signals have strong nonlinear characteristics (see Figure 2), using amplitude or frequency cannot ideally characterize such signals. To realize the feature extraction of complicated nonlinear signals, it is usually considered to use empirical mode decomposition (EMD) ${ }^{33}$ or wavelet transform ${ }^{34}$ to decompose a signal into multi-layer signals. Then a typical feature of each layer signal is extracted to form a preliminary feature matrix with high-dimensionality. Finally, principle component analysis (PCA) ${ }^{35}$ or manifold learning ${ }^{36,37}$ is used to reduce its dimensionality, obtaining a low-dimensional feature matrix. ${ }^{38-42}$ However, this typical feature extraction method has two limitations. ${ }^{43-46}$

1. The researchers should first analyze the acquired signals and then design a suitable feature that can distinguish the difference of the signals. Such a feature extraction method relies on signal processing techniques and diagnostic. It needs to be established under the good knowledge of the properties of the signals and abundant engineering experience. The designing processes not only increase time and economical costs but also consume much human labor.

2. Due to the complex structure of the mechanical equipment and the harsh operation environment, massive signals collected by the monitoring system are usually non-linear signals that contain multiple natural oscillatory modes, which means that single typical feature extraction method cannot characterize these signals. 
To overcome the above two shortcomings, a new feature extraction method that combined independent mode functions reconstruction and linear local tangent space alignment (IMFR-LLTSA) is proposed in this paper. Firstly, by using the ensemble empirical mode decomposition (EEMD) algorithm ${ }^{47}$ a set of independent mode functions (IMFs) are obtained. Then the first several IMFs containing the main information of the original signal are selected to reconstruct a high-dimensional sample matrix. Finally, by using LLTSA algorithm, ${ }^{48}$ a low-dimensional dataset is mapped from the obtained high-dimensional sample matrix to obtain an adaptive low-dimensional feature matrix. In this method, the self-adaptability and the anti-mode mixing of the EEMD are fully used. More importantly, by reconstructing the IMFs and with the help of the LLTSA, an adaptive lowdimensional feature matrix is obtained, in which the non-linear dimension reduction capability of manifold learning is fully used to excavate the inherent structures from high-dimensional datasets, thus avoiding the feature selection problem.

\section{Contribution and structure of this paper}

The goal of this work is to develop a method to diagnose the small-amplitude hunting of high-speed trains. The main contributions of this paper are summarized as follows:

1. By analyzing the train tracking experimental data, the authors found that when the train is in the hunting motion state, there are unstable phenomena in all lateral, longitudinal, and vertical sides of the bogie. Based on this, an idea of bogie frame's lateral-longitudinal-vertical data fusion is proposed.

2. Small-amplitude hunting signals have strong nonlinear characteristics; the typical feature extraction methods, usually, cannot accurately characterize such non-linear signals. Therefore, a new feature extracting method based on IMFR-LLTSA is proposed. This method utilizes the self-adaptability of EEMD and the excellent dimensionality reduction capability of LLTSA. The simulation experiment shows the correctness and superiority of the method.

3. A small-amplitude hunting motion diagnosis method combining the idea of bogie frame's lateral-longitudinal-vertical data fusion and IMFRLLTSA is proposed. This method has been validated using the experimental data of the CRH380a running on the Shanghai-Hangzhou line. The results show that this method is superior to the single lateral diagnosis method.

The rest of this paper is structured as follows: The next section proposes an idea of judging the small-amplitude hunting based on the bogie frame's lateral-longitudinal-vertical data fusion method. Then the theory of EEMD and LLTSA is presented and the IMFR-LLTSA feature extraction method is proposed. The effectiveness and advantages are verified by three simulated signals. Then, the idea of bogie frame's lateral-longitudinal-vertical data fusion and the IMFR-LLTSA feature extraction method are combined to identify the small-amplitude hunting of highspeed trains. This method is verified by a case study. Finally, conclusions are drawn in the last section.

\section{The idea of bogie frame's lateral-longitudinal-vertical data fusion method}

\section{Small-amplitude hunting definition}

When the hunting motion occurs on trains, the hunting information is shown not only in the bogie's lateral signal but also in the longitudinal and vertical signals (see Figure 3(b)). In addition, when the train transforms from the normal state to the standard hunting state, the small-amplitude hunting motion occurs easily. The small-amplitude hunting is also a sign of the standard hunting. According to the literature, ${ }^{18,22,30}$ referencing the frame's lateral acceleration signal, this paper defines the standard hunting, normal and small-amplitude hunting as follows:

- Standard hunting: the part of the bogie frame's lateral acceleration with the peak value reaching or exceeding the limit value $8 \mathrm{~m} / \mathrm{s}^{2}$ at more than six times (including the sixth time) continuously;

- Normal condition: the part of the bogie frame's lateral acceleration with the peak value no more than $2 \mathrm{~m} / \mathrm{s}^{2}$;

- Small-amplitude hunting: the part of the peak value of the bogie frame's lateral acceleration does not reach (or exceed) $8 \mathrm{~m} / \mathrm{s}^{2}$ for six consecutive times, but exceeds $2 \mathrm{~m} / \mathrm{s}^{2}$ many times and is seriously unstable.

\section{Analysis of bogie frame's lateral, longitudinal, and vertical signals}

From Figures 2 and 3(b), it can be known that:

- When the bogie is under the standard hunting condition, the amplitudes of bogie frame's lateral, longitudinal, and vertical acceleration signals are all larger than that under the other two conditions, showing extremely obvious unstable phenomena.

- When the bogie is under the normal running condition, its lateral, longitudinal, and vertical acceleration signals are all in a random vibration state, and the amplitudes are smaller than that in the standard hunting.

- When the bogie is under the small-amplitude hunting condition, the peak values of the lateral, 


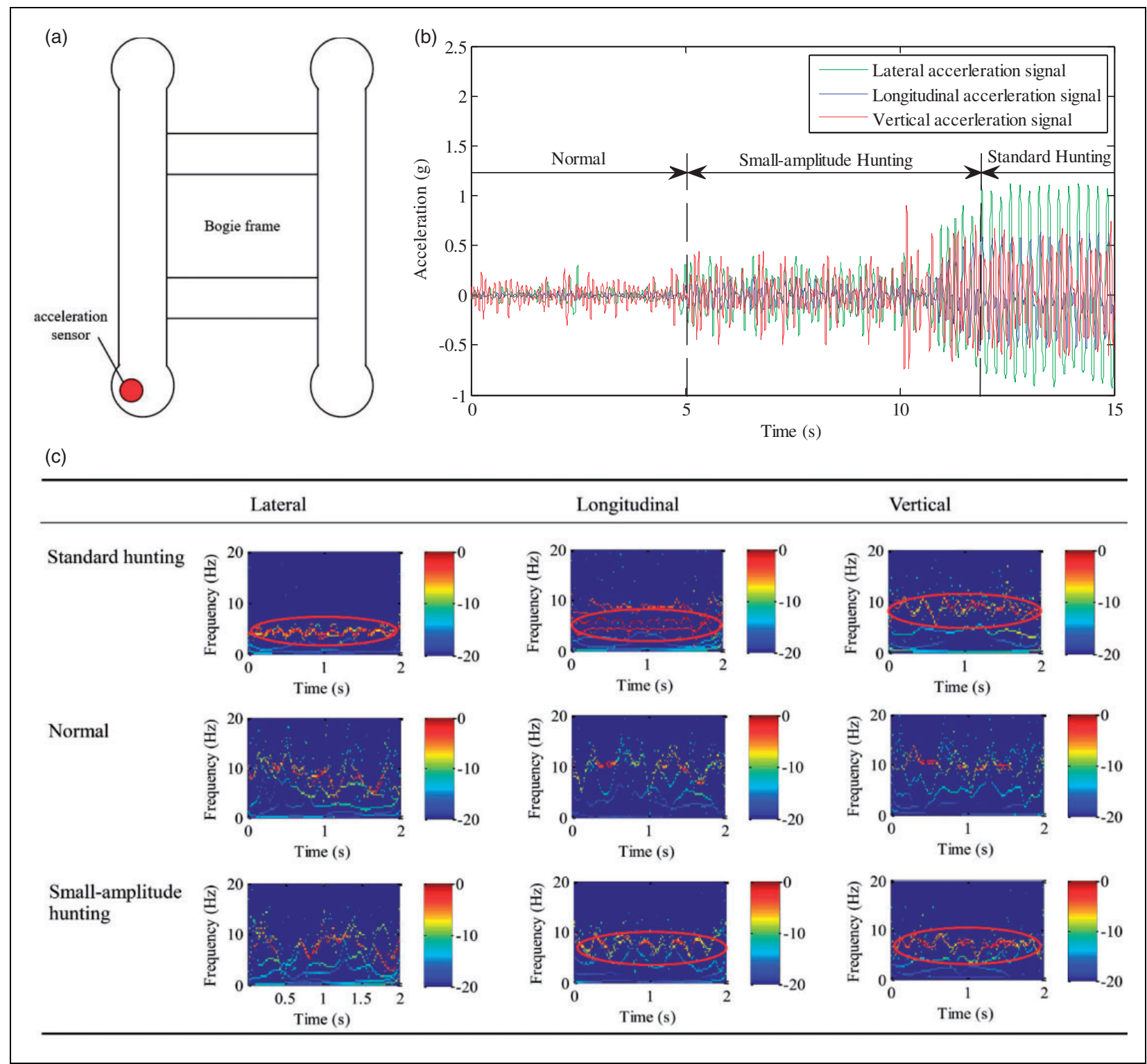

Figure 3. The measuring point position on the bogie frame (a), the acceleration signals of the bogie frame in three directions (lateral, longitudinal and vertical) during normal, small-amplitude hunting and standard hunting states (b), and their time-frequency distributions after HHT (c).

longitudinal, acceleration signals are lower than that under the standard hunting, and even the amplitudes of part of the signals are close to that under the normal state.

HHT is introduced to analyze the time-frequency characteristics of the signals, as shown in Figure 3(c). Obviously, in the standard hunting state, the instability information is characterized in all three directions, and the standard hunting can be easily distinguished by HHT. However, the use of only the lateral signal cannot ideally distinguish the normal and smallamplitude hunting, since the time-frequency characteristics in normal and small-amplitude hunting states are not obvious.

The reason is that the small-amplitude hunting, sometimes, is in the process of supercritical bifurcation, ${ }^{18,19}$ which means that it is the transition state from the normal state to the standard hunting. Therefore, the time-frequency characteristics of the small-amplitude hunting are close to that of the normal state in the early stages of the supercritical bifurcation and are close to that of the standard hunting before evolving into a standard hunting motion. Using the lateral signal to recognize the small-amplitude hunting, sometimes, is not ideal.

At that time, the longitudinal and vertical vibration signals contain a wealth of instability information of the small-amplitude hunting information (see the red ellipses in Figure 3(c)). Therefore, for achieving the small-amplitude hunting monitoring, an idea of bogie frame's lateral-longitudinal-vertical data fusion is proposed in this paper. The essence of this method is to make comprehensive use of the instability information distributed in the three directions of the bogie frame. The specific application steps are described in 
the section 'Small-amplitude hunting diagnosis method based on bogie frame's lateral-longitudinal-vertical data fusion and IMFR-LLTSA'.

\section{IMFR-LLTSA-based feature extraction method}

\section{Theory of EEMD}

EMD. EMD, which was proposed by Huang et al., is a time-frequency signal processing method that can self-adaptively decompose a nonlinear signal into a set of IMFs. ${ }^{49}$ Each IMF indicates a simple oscillation mode with a physical meaning. The definition and calculation steps of EMD are described as follows ${ }^{50}$ :

1. Identify all the local maxima of signal $x(t)$ and connect all the extrema by a cubic spline line as the upper envelope $m_{+}$. Repeat the procedure on the local minima of signal $x(t)$ to produce the lower envelope $m_{-}$and set the mean of $m_{+}$and $m_{-}$as $m_{1}$

$$
m_{1}=\frac{\left(m_{+}+m_{-}\right)}{2}
$$

2. Designate the difference between the signals $x(t)$ and $m_{1}$ as the first component, $h_{1}$ i.e.

$$
h_{1}=x(t)-m_{1}
$$

If $h_{1}$ meets the IMF's requirements given in Fan et al., ${ }^{51}$ take it as the first IMF of $x(t)$. If it does not satisfy the requirements, take it as the original signal and repeat the above steps until the component $h_{1 k}$ meets the requirements of a strict IMF, and $h_{1 k}$ is regarded as the first IMF $c_{1}$

$$
c_{1}=h_{1 k}
$$

3. Separate the first IMF $c_{1}$ from the $x(t)$ by

$$
r_{1}=x(t)-c_{1}
$$

4. Treat the $r_{1}$ as the original signal and subject it to the same above process. The decomposition is stopped when $r_{\mathrm{N}}$ is monotonous or lower than the predetermined value of substantial consequence. Then, the other $N-1$ IMFs can be achieved, which satisfy

$$
\left\{\begin{array}{c}
r_{1}-c_{2}=r_{2} \\
\vdots \\
r_{N-1}-c_{n}=r_{N}
\end{array}\right.
$$

5. By summing up equations (4) and (5), finally, the signal $x(t)$ can be decomposed as

$$
x(t)=\sum_{n=1}^{N} c_{n}(t)+r_{N}(t)
$$

EEMD. One shortcoming of EMD algorithm is the mode mixing; EEMD is proposed to alleviate the mode mixing problem of EMD using white noiseassisted analysis method. ${ }^{47}$ The EEMD process of signal $x(t)$ can be briefly described as follows:

1. Add white noise with a predefined amplitude to the original signal $x(t)$ to generate a new signal.

2. Use the EMD algorithm to decompose the new signal into several IMFs.

3. Repeat the above two steps $M$ times with different white noise, where the amplitude of the added white noise is fixed and $M$ is a positive integer presented.

4. Calculate the ensemble means of the corresponding IMFs achieved in the above $M$ decomposition trials by

$$
\left\{\begin{array}{l}
\bar{c}_{n}=\frac{1}{M} \sum_{m=1}^{M} c_{n, m} \\
\bar{r}_{N}=\frac{1}{M} \sum_{m=1}^{M} r_{N, m}
\end{array}\right.
$$

where $n=1,2, \ldots N, m=1,2, \ldots M$. Finally, the original signal can be decomposed as

$$
x(t)=\sum_{n=1}^{N} \bar{c}_{n}(t)+\bar{r}_{N}(t)
$$

\section{Theory of LLTSA}

Manifold learning. Manifold learning is a new dimensionality reduction tool. It can extract the intrinsic geometry information of a high-dimensional dataset, i.e., to excavate the embedded low-dimensional manifold hidden in the high-dimensional observation space without losing important information. ${ }^{36,37}$ It performs well in dealing with non-linear or non-stationary signals and has been applied in machinery fault diagnosis. ${ }^{40,52}$ Currently, classical manifold learning algorithms include ISOMAP, ${ }^{36}$ locally linear embedding (LLE), ${ }^{37}$ local tangent space alignment (LTSA) ${ }^{53}$ LLTSA,${ }^{54}$ etc. Among those algorithms, LLTSA can minimize the cost function of LTSA algorithm linearly. Therefore, this paper uses the LLTSA algorithm.

LLTSA. Suppose a high-dimensional dataset $\mathbf{X}=$ $\left\{\mathbf{x}_{i} \in \mathbf{R}^{D}, i=1,2, \ldots, N\right\}$, which is sampled from an underlying manifold belonging to a lower dimensional feature space in $\mathbf{R}^{d}$, where $d<D$. The dimensionality reduction problem of LLTSA then can be considered to find out a projection matrix $\mathbf{A}$ to map the highdimensional dataset $\mathbf{X}$ into a low-dimensional dataset $\mathbf{Y}=\left\{\mathbf{y}_{i} \in \mathbf{R}^{D}, i=1,2, \ldots N\right\}$ as

$$
\mathbf{Y}=\mathbf{A}^{\mathrm{T}} \mathbf{X H}_{N}
$$

where $\mathbf{H}_{N}=\mathbf{I}-\mathbf{e e}^{\mathrm{T}} / N$. The basic assumption of LLTSA is that the local structure information in the 
neighborhood of each sample can be expressed by its local tangent space. Thus, the local tangent spaces of all samples can be realigned in the global low-dimensional feature space to construct the projection matrix from high-dimensional input space to the low-dimensional feature space.

In the LLTSA algorithm, the neighborhood $\mathbf{X}_{i}=$ $\left[\mathbf{x}_{i 1}, \mathbf{x}_{i 2}, \ldots, \mathbf{x}_{i k}\right]$ of sample point $\mathbf{x}_{i},(i=1,2, \ldots, N)$ is constructed according to the Euclidean distance between samples. Then, a local transformation matrix $\mathbf{Q}_{i}$ is needed to map the neighborhood $\mathbf{X}_{i}$ to the local low-dimensional tangent space. Therefore, the local structure of the neighborhood of sample point $\mathbf{x}_{i}$ can be approximately expressed as

$$
\begin{aligned}
& \arg \min _{\mathbf{x}, \mathbf{\Theta}, \mathbf{Q}} \sum_{j=1}^{k}\left\|\mathbf{x}_{i j}-\left(\mathbf{x}+\mathbf{Q}_{i} \theta_{i j}\right)\right\|_{2}^{2} \\
& =\arg \min _{\mathbf{\Theta}, \mathbf{Q}} \sum_{j=1}^{k}\left\|\mathbf{X}_{i} \mathbf{H}_{k}-\mathbf{Q}_{i} \Theta_{i}\right\|_{2}^{2}
\end{aligned}
$$

In equation (10), $\theta_{i j}$ denotes the local low-dimensional representation of the nearest neighbor $x_{i j}$, $\Theta_{i}=\left[\theta_{i 1}, \theta_{i 2}, \ldots, \theta_{i k}\right]$ represents the local low-dimensional representation of $\mathbf{X}_{i}$, and $\mathbf{H}_{k}=\mathbf{I}-\mathbf{e e}^{\mathrm{T}} / k$ is the centering matrix. Obviously, the optimal $\mathbf{x}$ can be given by the average value of all $\mathbf{x}_{i j}$, and the optimal transformation matrix $\mathbf{Q}_{i}$ can be given by the $d$ eigenvectors of covariance matrix $\left(\mathbf{X}_{i} \mathbf{H}_{k}\right)\left(\mathbf{X}_{i} \mathbf{H}_{k}\right)^{\mathrm{T}}$ corresponding to its maximal eigenvalues. Therefore, $\Theta_{i}$ can be calculated through $\Theta_{i}=\mathbf{Q}_{i}^{\mathrm{T}} \mathbf{X}_{i} \mathbf{H}_{k}$. Therefore, it can be considered that LLTSA performs a local PCA on $\mathbf{X}_{i}$ to approximate the local structure in the neighborhood.

After obtaining the local structure, the local tangential spaces of all samples are rearranged in the global low-dimensional feature space to obtain the global low-dimensional representation $\mathbf{Y}$ of $\mathbf{X}$. The selection matrix is $\mathbf{S}=\left[\mathbf{S}_{1}, \mathbf{S}_{2}, \ldots, \mathbf{S}_{N}\right]$ among which $\mathbf{S}_{i},(i=1,2, \ldots, N)$ is a $0-1$ selection vector, so that $\mathbf{Y}_{i}=\mathbf{Y S}_{i}$ in which $\mathbf{Y}_{i}=\left[\mathbf{y}_{i 1}, \mathbf{y}_{i 2} \ldots, \mathbf{y}_{i k}\right]$ is the global low-dimensional representation of $\mathbf{X}_{j}$. The objective function of this step can be converted into the following minimum problem

$$
\begin{aligned}
& \arg \min _{\mathbf{Y}} \sum_{i=1}^{N}\left\|\mathbf{E}_{i}\right\|^{2}=\arg \min _{\mathbf{Y}} \sum_{i=1}^{N}\left\|\mathbf{Y}_{i} \mathbf{H}_{k}-\mathbf{L}_{i} \Theta_{i}\right\|^{2} \\
& =\arg \min _{\boldsymbol{Y}}\|\mathbf{Y S W}\|_{2}^{2}=\arg \min _{\mathbf{Y}} \operatorname{tr}\left(\mathbf{Y S W W} \mathbf{W}^{\mathrm{T}} \mathbf{S}^{\mathrm{T}} \mathbf{Y}^{\mathrm{T}}\right)
\end{aligned}
$$

where $\mathbf{L}_{i}$ denotes a global transformation matrix and the optimal $\mathbf{L}_{i}$ is given by $\mathbf{L}_{i}=\mathbf{Y}_{i} \mathbf{H}_{k} \Theta_{i}^{\dagger}$, in which $\Theta_{i}^{\dagger}$ is the Moore-Penrose generalized inverse of $\Theta_{i} ; \mathbf{W}=$ $\operatorname{diag}\left(\mathbf{W}_{1}, \mathbf{W}_{2}, \ldots, \mathbf{W}_{N}\right)$, where $\mathbf{W}_{i}$ is given by $\mathbf{W}_{i}=$ $\mathbf{H}_{k}\left(\mathbf{I}-\Theta_{i} \Theta_{i}^{\mathrm{T}}\right)$. Taking the objective of LLTSA in equation (9) into account, equation (11) can be converted to

$$
\left\{\begin{array}{l}
\underset{\arg \min }{\operatorname{tr}}\left(\mathbf{A}^{\mathrm{T}} \mathbf{X} \mathbf{H}_{N} \mathbf{B} \mathbf{H}_{N} \mathbf{X}^{\mathrm{T}} \mathbf{A}\right) \\
\mathbf{A}^{\mathrm{T}} \mathbf{X H}_{N} \mathbf{B} \mathbf{H}_{N} \mathbf{X}^{\mathrm{T}} \mathbf{A}=\mathbf{I}_{d}
\end{array}\right.
$$

where $\mathbf{B}=\mathbf{S W} \mathbf{W}^{\mathrm{T}} \mathbf{S}^{\mathrm{T}} . \mathbf{A}^{\mathrm{T}} \mathbf{X} \mathbf{H}_{N} \mathbf{B} \mathbf{H}_{N} \mathbf{X}^{\mathrm{T}} \mathbf{A}=\mathbf{I}_{d}$ is applied to uniquely determine $\mathbf{Y}$. Finally, the solution of equation (10) can be transformed into a generalized eigenvalue problem as

$$
\mathbf{A}^{\mathrm{T}} \mathbf{X} \mathbf{H}_{N} \mathbf{B} \mathbf{H}_{N} \mathbf{X}^{\mathrm{T}} \boldsymbol{\alpha}=\lambda \mathbf{X} \mathbf{H}_{N} \mathbf{X}^{\mathrm{T}} \boldsymbol{\alpha}
$$

The transformation matrix A can be given by the $d$ eigenvectors $\boldsymbol{\alpha}_{1}, \boldsymbol{\alpha}_{2}, \ldots, \boldsymbol{\alpha}_{d}$ corresponding to the eigenvalues $\lambda_{1} \leqslant \lambda_{2} \leqslant \cdots \leqslant \lambda_{d}$ of equation (13). That is to say $\mathbf{A}=\boldsymbol{\alpha}_{1}, \boldsymbol{\alpha}_{2}, \ldots, \boldsymbol{\alpha}_{d}$.

\section{IMFR-LLTSA feature extraction method}

Theory of IMFR-LLTSA. In fault diagnosis, feature selection is a difficult issue, especially for the non-linear signals that often appear in the machinery field. Using time-domain features or frequency-domain features to identify such signals with strong non-linear characteristics, usually, cannot achieve the desired result. Therefore, EEMD has been widely used in dealing with such non-stationary signals due to its self-adaptability. This method is used to decompose a signal into a group of IMFs. Then, the typical features of the IMFs are extracted to build a feature matrix with a high dimensionality. In the face of the high-dimensional feature dataset, dimensionality reduction tools such as PCA and manifold learning are usually applied.

However, this typical feature extraction method has two limitations: (a) It needs to be established under the condition that the signal is extremely well understood. Then we can know which feature can best reflect the properties of the signals. The designing processes not only increase time and economical costs but also consume much human labor. $^{44}$ (b) Such a typical feature extraction method only considers one single aspect information of the signals. To some extent, some important fault information contained in the signal may be lost. It often fails to achieve an ideal result in the face of mechanical equipment with severe fault information coupling. ${ }^{43}$

To solve the above two shortages, an adaptive feature extraction method based on IMFR-LLTSA is proposed in this paper. In this method, the original signal is decomposed through EEMD, and the signal information is spread out on multiple scales. The signal for each scale is then reconstructed directly. This reconstructed high-dimensional matrix contains the original information for each scale signal. Finally, LLTSA is used to reduce the dimensionality of the high-dimensional matrix, obtaining a low-dimensional structure, and the resulting low-dimensional structure is the final adaptive feature. In this method, the self-adaptability of EEMD and the excellent dimensionality reduction ability of LLTSA are utilized, avoiding the feature 
selection problem. The specific steps are described as follows:

1. EEMD: For the time series $x(t)$, a group of IMFs are obtained through EEMD.

$$
x(t)=\sum_{n=1}^{N} \bar{c}_{n}+\bar{r}_{N}
$$

2. After obtaining the IMFs, the first $q$, IMFs containing the main information are selected to construct a new function $\mathbf{I M F}_{\text {new }}$ according to the following formula

$$
\mathbf{I M F}_{\text {new }}=\left[\bar{c}_{1} \bar{c}_{2} \bar{c}_{3} \ldots \bar{c}_{q}\right]
$$

3. Steps 1 and 2 are applied to all samples, and a sample matrix $\mathbf{S}$ is built.

$$
\mathbf{S}=\left[\begin{array}{c}
\bar{c}_{11} \bar{c}_{12} \bar{c}_{13} \ldots \bar{c}_{1 q} \\
\bar{c}_{21} \bar{c}_{22} \bar{c}_{23} \ldots \bar{c}_{2 q} \\
\vdots \\
\bar{c}_{p 1} \bar{c}_{p 2} \bar{c}_{p 3} \ldots \bar{c}_{p q}
\end{array}\right]
$$

4. Dimensionality reduction through the manifold learning: LLTSA is used to reduce the dimensionality of the sample matrix $\mathbf{S}$ for obtaining a lowdimensional feature matrix, as shown in the following formula

$$
\mathbf{S} \stackrel{\text { LLTSA }}{\rightarrow}\left[\mathbf{f}_{1}, \mathbf{f}_{2}, \ldots, \mathbf{f}_{d}\right]
$$

5. Training and recognition: The low-dimensional manifold features calculated by the above steps are classified into training samples and test samples. Then, LSSVM is used to realize the state identification.

Signal simulation. To verify the performance of the IMFR-LLTSA feature extraction method, three signals are simulated according to the literature. ${ }^{55}$

$$
\left\{\begin{array}{l}
a(t)=-1.2154 w_{1}(t)+0.6157 w_{2}(t)+n_{1}(t) \\
b(t)=-1.4840 w_{1}(t)+0.3891 w_{2}(t)+n_{2}(t) \\
c(t)=-1.6839 w_{1}(t)+0.1035 w_{2}(t)+n_{3}(t)
\end{array}\right.
$$

where $\quad w_{1}(t)=[1+0.8 \cos (0.6 \pi t)] \cdot \sin (6 \pi t), \quad w_{2}(t)=$ $[1+0.8 \cos (0.6 \pi t)] \cdot \sin (6.2 \pi t) . n_{1}(t), n_{2}(t)$ and $n_{3}(t)$ are the Gaussian white noise (when SNR $=0.2$ ). The sampling frequency is $20 \mathrm{~Hz}$.

As plotted in Figure 4(a), the amplitudes of these three simulated signals at the red arrows are different, but the difference is not obvious. FFT and HHT results of the three simulated signals are plotted in
Figure 4(b) and (c), respectively. Obviously, it is very hard to distinguish the three signals by using simple time-frequency analysis methods.

Feature extraction and clustering evaluation. To solve this problem, the IMFR-LLTSA is thus introduced. Sixty samples for each type of signal are selected for a total of 180 . The data length $N$ of each sample is set to 400 , and the time length is $20 \mathrm{~s}$. All samples are decomposed by EEMD, and the EEMD results for one sample of $a(t), b(t)$ and $c(t)$ are shown in Figure 4(d).

After processing the original signal through EEMD, the selection of the IMF components is also important. Since EEMD is a principal component analysis method, the primary information of the original signal is included in the first several IMFs. In this research, the number of IMFs of all the samples is greater than 6. Also, we calculated the energy of the first six IMFs and found that it is always greater than $94.32 \%$ of the total energy: $\left(\sum_{i=1}^{6} E_{i}\right) / E_{s} \geqslant 94.32 \%$. Therefore, to ensure the subsequent processing of the IMFR-LLTSA method, and to retain the information to the greatest extent, the first six IMFs of each sample are selected to be reconstructed to form a $180 \times 2400$ highdimensional feature matrix.

As the section LLTSA indicates, in the LLTSA algorithm, $k$ and $d$ need to be determined. In this study, we set $k=7$ and $d=3$. Then the dimensionality is reduced to a $180 \times 3$ low-dimensional feature matrix by using the LLTSA method (see Figure 5(a)). It can be seen from the figure that the three simulated signals can be completely classified through LLTSA. For demonstrating the superiority of the LLTSA, PCA is introduced. The clustering result is shown in Figure 5(b). The comparison result shows that the IMFR-PCA can classify the three simulated signals, but its clustering result is significantly worse than that of IMFR-LLTSA.

To demonstrate that the IMFR-LLTSA method is superior to the EEMD-typical feature-LLTSA method, the energy and SampEn features that are commonly applied in machinery are introduced for simulation analysis. The energy and Sample entropy (SampEn) features of the first six IMFs are extracted to construct a $180 \times 6$ high-dimensional feature matrix. Then the feature matrix is reduced by LLTSA to generate a $180 \times 3$ low-dimensional feature matrix (see Figure 5(c) and (d)). It can be known that EEMD-energy-LLTSA can separate the three classes of signals, but the clustering result is significantly worse than that of IMFR-LLTSA, and EEMDSampEn-LLTSA method cannot even separate those three simulated signals.

To accurately evaluate the clustering ability of these methods, three evaluation indices, within-class scatter $S_{w}$, between-class scatter $S_{b}$, and the ratio $S_{w} / S_{b}$, are introduced to evaluate the clustering result. ${ }^{41}$ 


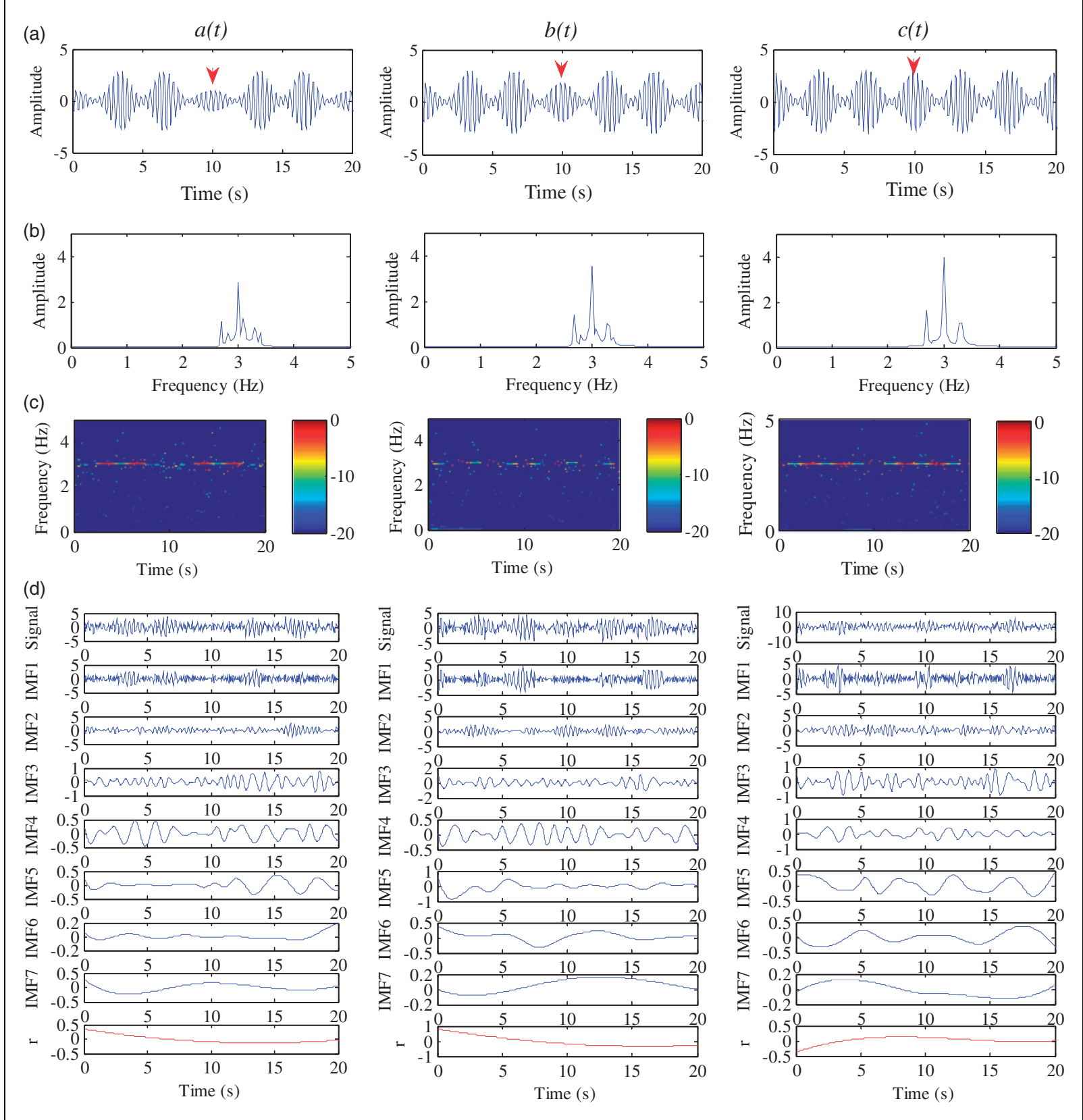

Figure 4. Time-frequency characteristics of three simulated signals: (a) time domain, (b) frequency domain, (c) time-frequency distribution after HHT, and (d) EEMD results.

For a feature matrix $\left[\mathbf{f}_{1}, \mathbf{f}_{2}, \ldots, \mathbf{f}_{d}\right]$, the two parameters, $S_{w}$ and $S_{b}$, are defined as

$$
\left\{\begin{array}{l}
S_{w}=\sum_{p=1}^{c} \sum_{f_{k} \in C_{p}}\left(f_{k}-\mu_{f}^{p}\right)\left(f_{k}-\mu_{f}^{p}\right)^{T} \\
S_{b}=\sum_{p=1}^{c}\left(\mu_{f}^{p}-\bar{\mu}_{f}\right)\left(\mu_{f}^{p}-\bar{\mu}_{f}\right)^{T}
\end{array}\right.
$$

where $\mu_{f}^{p}$ denotes the mean of the eigenvector for a sample with a given class $C_{p}(p=1,2, \ldots, c), c$ denotes the number of the classification, and $\bar{\mu}_{f}=1 / c \sum_{p=1}^{c} \mu_{f}^{p}$ represents the total mean of the eigenvectors of all classes. $S_{w}$ denotes the compact degree of distribution of each class of sample, $S_{b}$ denotes the degree of separation of different classes, and $S_{w} / S_{b}$ denotes the overall clustering effect, where the smaller value indicates that the clustering effect is better.

The accurate evaluation of the features achieved by the above methods is listed in Table 1. The results show clearly that the IMFR-LLTSA method has good capabilities of both classification and clustering and is superior to the IMFR-PCA and EEMDLLTSA methods. Because in the feature extraction process, the typical single feature extraction method only considers one single aspect information of signals. To some extent, it loses other useful information contained in the signal. This typical feature extraction method often fails to achieve desired results in the face of mechanical equipment with severe fault 


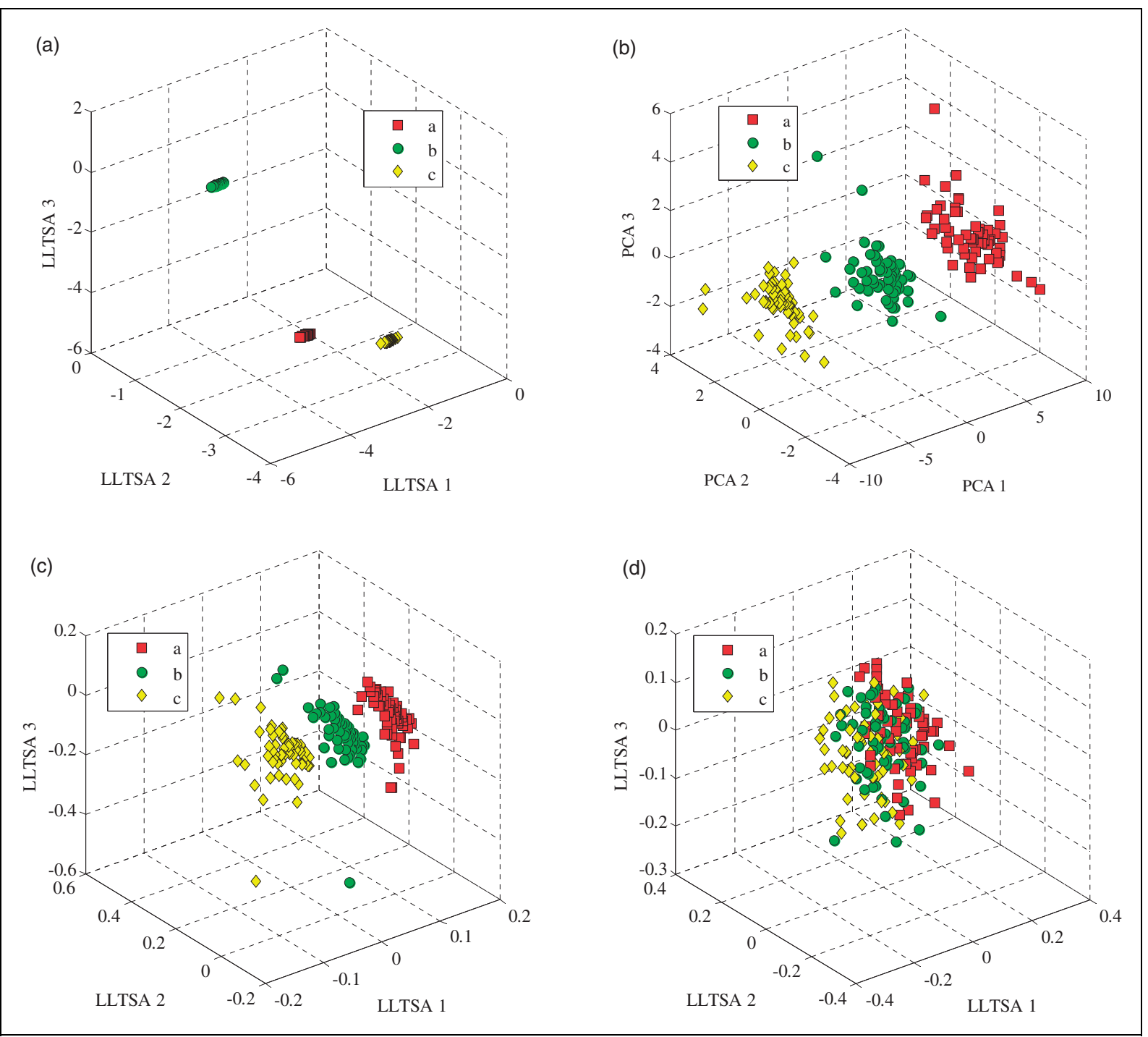

Figure 5. Clustering effect of different methods: (a) IMFR-LLTSA, (b) IMFR-PCA, (c) EEMD-energy-LLTSA, and (d) EEMD-SampEnLLTSA.

Table I. Clustering evaluation of different features for the three signals.

\begin{tabular}{lllrr}
\hline & & \multicolumn{2}{c}{ EEMD-LLTSA } \\
\cline { 5 - 5 } & IMFR-LLTSA & IMFR-PCA & \multicolumn{1}{c}{ Energy } & \multicolumn{1}{c}{ SampEn } \\
\hline Within-class scatter $S_{w}$ & 0.1633 & 1.2213 & 2.0081 & 2.6076 \\
Between-class scatter $S_{b}$ & 2.1655 & 0.3869 & 0.0167 & 0.0065 \\
$S_{w} / S_{b}$ & $7.5410 \times 10^{-2}$ & 3.1566 & 120.2455 & 401.1692 \\
\hline
\end{tabular}

information coupling. However, in the proposed IMFR-LLTSA method, by directly reconstructing the IMFs, the reconstructed high-dimensional matrix contains the original information for each scale signal. Then, the non-linear dimension reduction capability of manifold learning is fully used to excavate the inherent structures from high-dimensional datasets. The resulting low-dimensional structure is regarded as the final adaptive feature, thus avoiding the feature selection problem.

\section{Small-amplitude hunting diagnosis method based on the bogie frame's lateral-longitudinal-vertical data fusion and IMFR-LLTSA}

Experimental operating conditions and signal acquisition

The experimental data, used in this paper, were from a scientific experiment for a CRH 380a high-speed train 
running on the Shanghai-Hangzhou railway line. The CRTS II non-ballasted track and seamless rail were used in the whole line. The measuring system (see Figure 6(a)) consists of the data acquisition unit, interchanger, vehicle-mounted computer, $3 \mathrm{G}$ router, and GPS speed measurement module. As the terminal control unit, the vehicle-mounted computer controlled the entire measuring system through the interior local area network (LAN). The signal acquisition unit acquired the vibration signals from sensors. The signals then were sent to the vehicle-mounted computer by the ZigBee wireless network system and the interchanger. The GPS module was used to measure the train speed and gave the feedback of the operation time. Then the speed and time information were sent to the vehicle-mounted computer by a $3 \mathrm{G}$ router. The acceleration signals used in this work were provided by an LC0709A-18 tri-axial acceleration sensor installed on the bogie frame (see Figure 6(b)), and the accuracy was $0.5 \%$. The original sampling frequency was $2500 \mathrm{~Hz}$. Figure 6(a) and (b) is taken from literature, ${ }^{31}$ as the monitoring system used in this paper is identical to the monitoring system in Zhai et al. ${ }^{31}$
Because the frequency band of hunting motion is $2-12.07 \mathrm{~Hz},{ }^{22}$ according to Shannon sampling theorem and engineering experience, the resampling frequency is selected as $250 \mathrm{~Hz}$. Then, $2-12.07 \mathrm{~Hz}$ bandpass filtering is conducted. The time-speed signal and time-acceleration signals of the bogie frame for a trip are shown in Figure 6(c). When the speed of the experimental train was $330-350 \mathrm{~km} / \mathrm{h}$, the smallamplitude hunting occurred repeatedly.

The selection of the sample length is also a question worth exploring. On the one hand, if the sample length is too long, the sampling time required will be longer; on the other hand, if the sample length is too short, the information contained in the sample is not enough. By observing the experimental data and referring to the literature, ${ }^{23,32}$ the time length is selected as $2 \mathrm{~s}$, with the sample length of 500 data points.

\section{Small-amplitude hunting motion diagnosis method based on bogie frame's single-lateral method and IMFR-LLTSA}

In order to verify that the IMFR-LLTSA method is suitable for small-amplitude hunting diagnosis, the

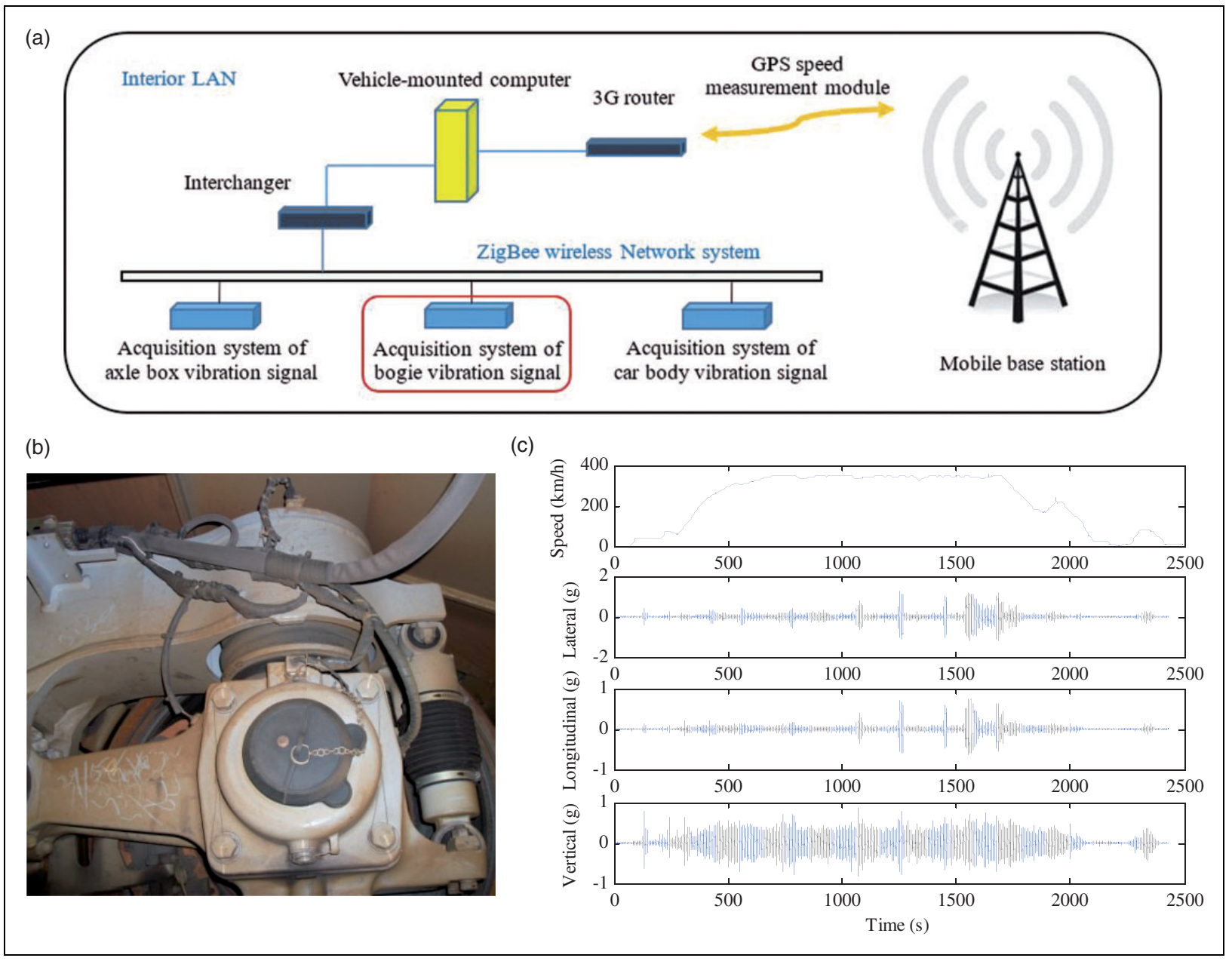

Figure 6. The measuring system of the CRH380a high-speed train (a), the installed acceleration sensor (b) and time-speed of the train and time-acceleration signals of the bogie frame $(c)$. 
single lateral acceleration signal is introduced. Firstly, 30 samples of the normal, small-amplitude hunting and standard hunting of the lateral signal are selected according to the section 'IMFR-LLTSA feature extraction method', for a total of 90 samples. According to the IMF selection principle described in the section 'Signal simulation', the first six IMFs are selected for reconstruction. After that, a $90 \times 3000$ high-dimensional feature matrix is established. Then the highdimensional feature matrix is reduced to a $90 \times 3$ low-dimensional feature matrix through the LLTSA algorithm, as shown in Figure 7(a). The experimental result shows that except for some samples of the normal and small-amplitude hunting that cannot be fully clustered, the standard hunting can be accurately classified, and the overall clustering effect is good. PCA is introduced as a comparison (see Figure 7(b)); the experimental result shows that its clustering effect is worse than that of the LLTSA. It is proved that the IMFR-LLTSA feature extraction method is suitable for monitoring the small-amplitude hunting and is superior to the IMFR-PCA method.

In order to demonstrate that this method is more suitable for small-amplitude hunting diagnosis than the traditional EEMD-typical feature-LLTSA method, the energy and SampEn are introduced. First, the signals are decomposed by EEMD. Then, the energy and SampEn features of the first six IMFs are extracted to build a $90 \times 6$ feature matrix. Finally, the dimensionality reduction is performed through LLTSA for obtaining a $90 \times 3$ low-dimensional matrix (see Figure 7 (c) and (d)). In contrast, the clustering effects of the EEMD-LLTSA are poor. The experiment results show that the IMFR-LLTSA method is suitable for extracting features of small-amplitude hunting and is superior to the EEMD-typical feature-LLTSA method.

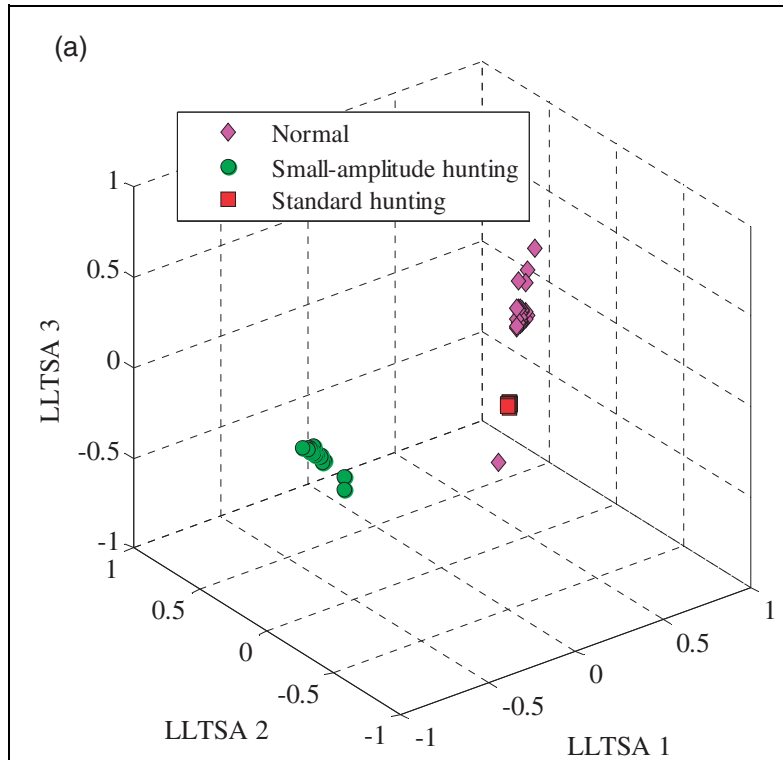

(c)

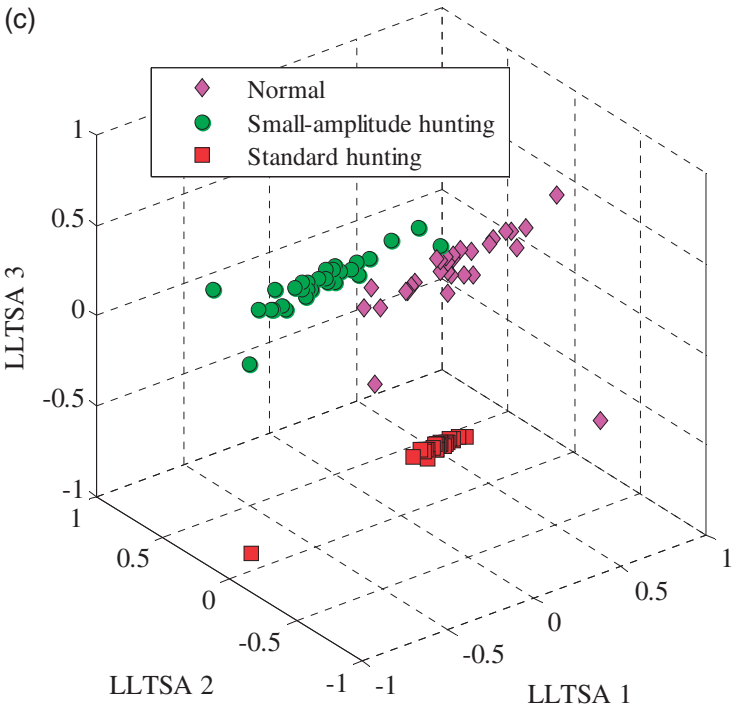

(b)

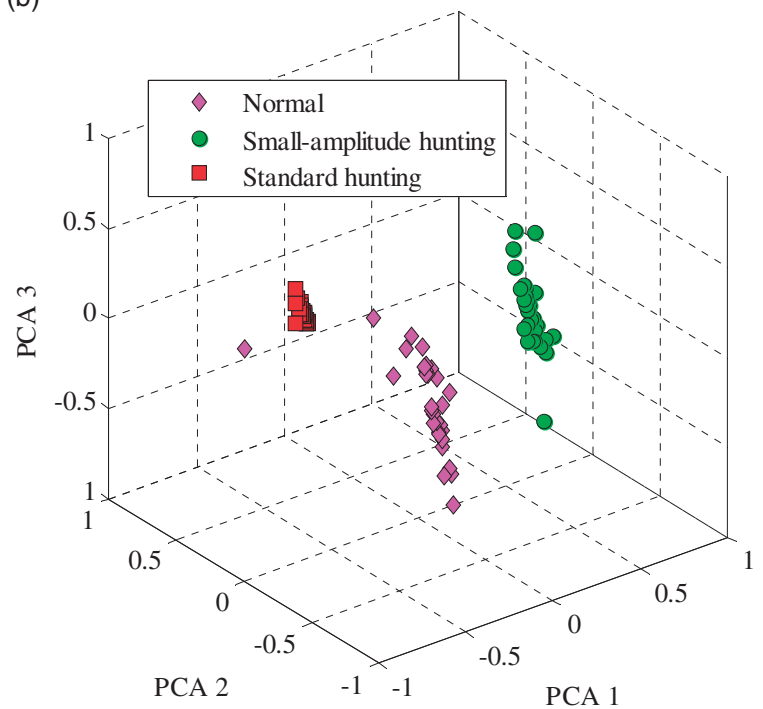

(d)

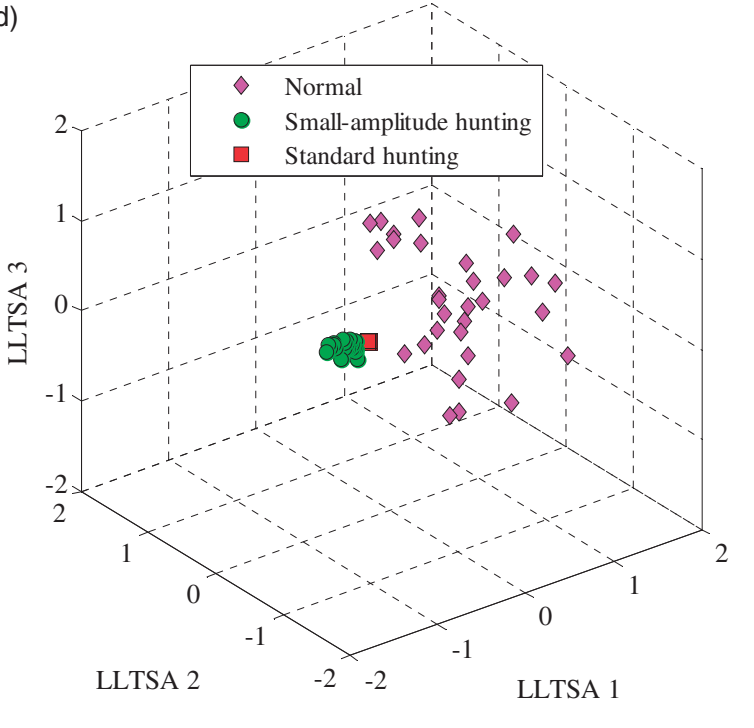

Figure 7. Results of IMFR-LLTSA (a), IMFR-PCA (b), EEMD-energy-LLTSA (c), and EEMD-SampEn-LLTSA (d). 


\section{Small-amplitude hunting motion diagnosis method based on the bogie frame's lateral-longitudinal- vertical data fusion and IMFR-LLTSA}

Technical route of bogie frame's lateral-longitudinal-vertical data fusion and IMFR-LLTSA. The traditional methods use only the lateral signal to monitor the stability of trains, which have omitted the longitudinal and vertical instability information. Therefore, in this section, an idea of bogie frame's lateral-longitudinal-vertical data fusion proposed in the section 'The idea of bogie frame's lateral-longitudinal-vertical data fusion method' and the IMFR-LLTSA proposed in the section 'IMFRLLTSA-based feature extraction method' are combined to identify the small-amplitude hunting. The technical route is shown in Figure 8, and the steps are as follows.

1. The EEMD is used to decompose the bogie frame's lateral, longitudinal, and vertical vibration signals to obtain the IMFs.

$\left\{\begin{array}{l}x_{\mathrm{La}}(t)=\sum_{n=1}^{N} \bar{c}_{\mathrm{La}, n}+\bar{r}_{\mathrm{La}, N} \\ x_{\mathrm{Lo}}(t)=\sum_{n=1}^{N} \bar{c}_{\mathrm{Lo}, n}+\bar{r}_{\mathrm{Lo}, N} \\ x_{\mathrm{Ve}}(t)=\sum_{n=1}^{N} \bar{c}_{\mathrm{Ve}, n}+\bar{r}_{\mathrm{Ve}, N}\end{array}\right.$
2. According to Figure 8, the IMF reconstruction is performed on the lateral, longitudinal, and vertical signals.

$$
\left\{\begin{array}{l}
\mathbf{I M F}_{\mathrm{La}, \text { new }}=\left[\bar{c}_{\mathrm{La}, 1} \bar{c}_{\mathrm{La}, 2} \ldots \bar{c}_{\mathrm{La}, n}\right] \\
\mathbf{I M F}_{\mathrm{Lo}, \text { new }}=\left[\bar{c}_{\mathrm{Lo}, 1} \bar{c}_{\mathrm{Lo}, 2} \ldots \bar{c}_{\mathrm{Lo}, n}\right] \\
\mathbf{I M F}_{\mathrm{Ve}, \text { new }}=\left[\bar{c}_{\mathrm{Ve}, 1} \bar{c}_{\left.\mathrm{Ve}, 2 \ldots \bar{c}_{\mathrm{Ve}, n}\right]}\right]
\end{array}\right.
$$

3. The normalization is performed on the above three vectors, and then the normalized three vectors are reconstructed into a new vector $\mathbf{I M F}_{\mathrm{LLV}}$.

$$
\begin{aligned}
\mathbf{I M F}_{\mathrm{LLV}} & =\left[\mathbf{I M F}_{\mathrm{La}, \text { new }} \mathbf{I M F}_{\mathrm{Lo}, \text { new }} \mathbf{I M F}_{\mathrm{Ve}, \text { new }}\right] \\
& =\left[\bar{c}_{\mathrm{La}, 1} \ldots \bar{c}_{\mathrm{La}, n} \bar{c}_{\mathrm{Lo}, 1} \ldots \bar{c}_{\mathrm{Lo}, n} \bar{c}_{\mathrm{Ve}, 1} \ldots \bar{c}_{\mathrm{Ve}, n}\right]
\end{aligned}
$$

4. Steps (1) to (3) are conducted to all samples to build the sample matrix $\mathbf{S}_{\mathrm{LLV}}$.

5. Dimensionality reduction and feature extraction through LLTSA: Use the LLTSA to reduce the dimensionality of the new vector $\mathbf{S}_{\mathrm{LLV}}$ obtained in Step (4) and extract the low-dimensional feature.

$\mathbf{S}_{\mathrm{LLV}} \stackrel{\text { LLTSA }}{\rightarrow}\left[\mathbf{f}_{1}, \mathbf{f}_{2}, \ldots, \mathbf{f}_{d}\right]$

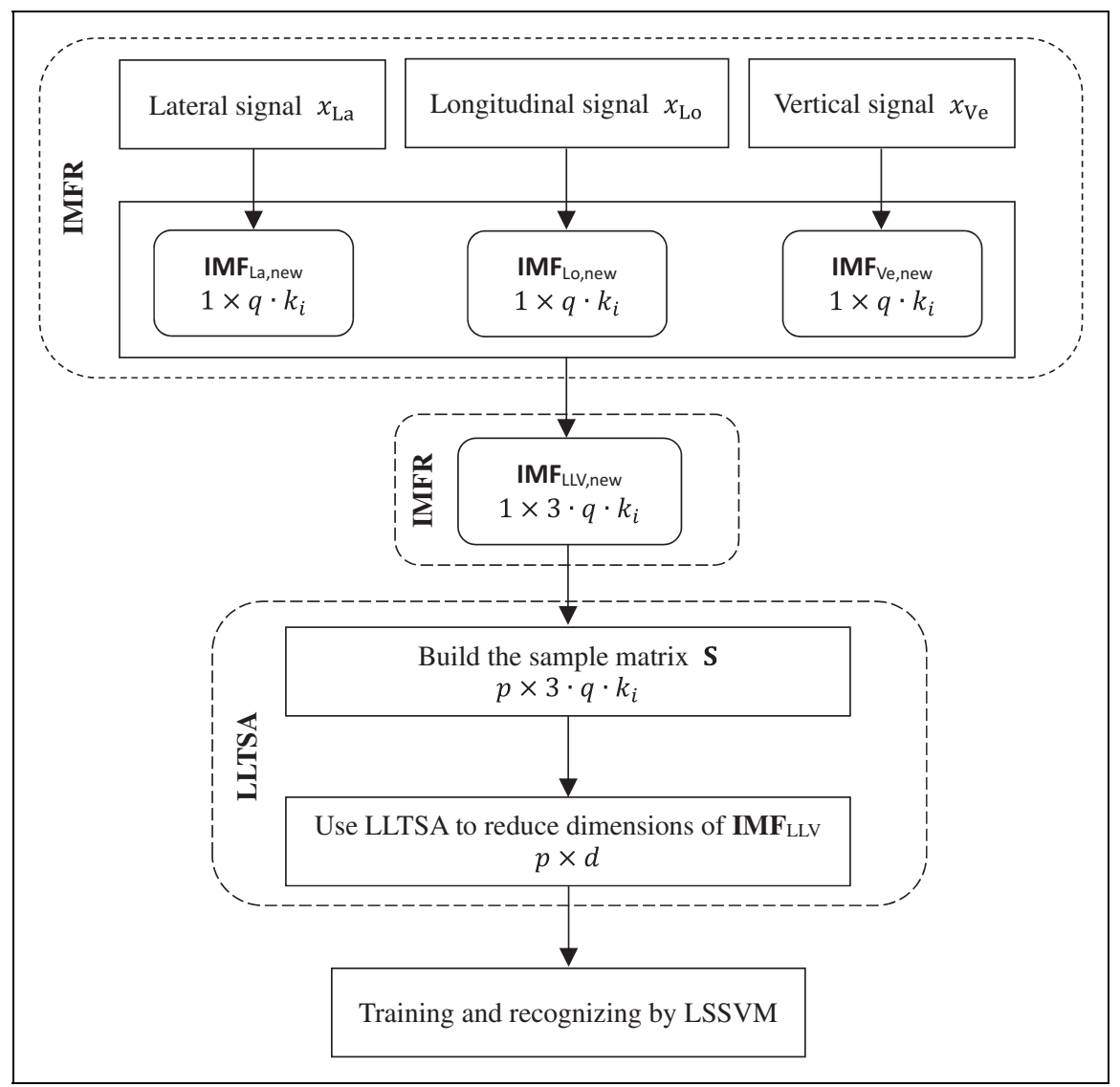

Figure 8. Technical route of combining the bogie frame's lateral-longitudinal-vertical data fusion and IMFR-LLTSA. 
Analysis of the method of integrating longitudinal, lateral and vertical signals. According to the technical route in Figure 8 , based on the IMFR-LLTSA method, the lateral-longitudinal-vertical, lateral-longitudinal, longitudinal-vertical, and lateral-vertical signals are respectively introduced, and the effects are shown in Figure 9. It shows that based on the IMFR-LLTSA, the separation of the three states can be realized by using lateral-longitudinal-vertical data fusion (see Figure 9(a)) and lateral-longitudinal data fusion (see Figure 9(b)) methods. However, the clustering effect obtained by the lateral-longitudinal-vertical data fusion method is the best, followed by the laterallongitudinal data fusion method (see Figure 9(b)), longitudinal-vertical data fusion method (see Figure 9(c)), and finally the lateral-vertical data fusion method (see Figure 9(d)). For precise evaluation, $S_{w}, S_{b}$, and $S_{w} / S_{b}$ are also calculated as listed in Table 2. The results show that the clustering capability of IMFR-LLTSA method is the best.

\section{Identification of LSSVM}

LSSVM is used to train and identify the low-dimensional features obtained in the previous two sub-sections. In total, 45 groups (15 groups normal, 15 groups small-amplitude hunting and 15 groups standard hunting) are used for training, and another 45 groups (15 groups normal, 15 groups small-amplitude hunting and 15 groups standard hunting) are used for testing. The results are shown in Table 2. By comparison, the method of the lateral-longitudinal-vertical
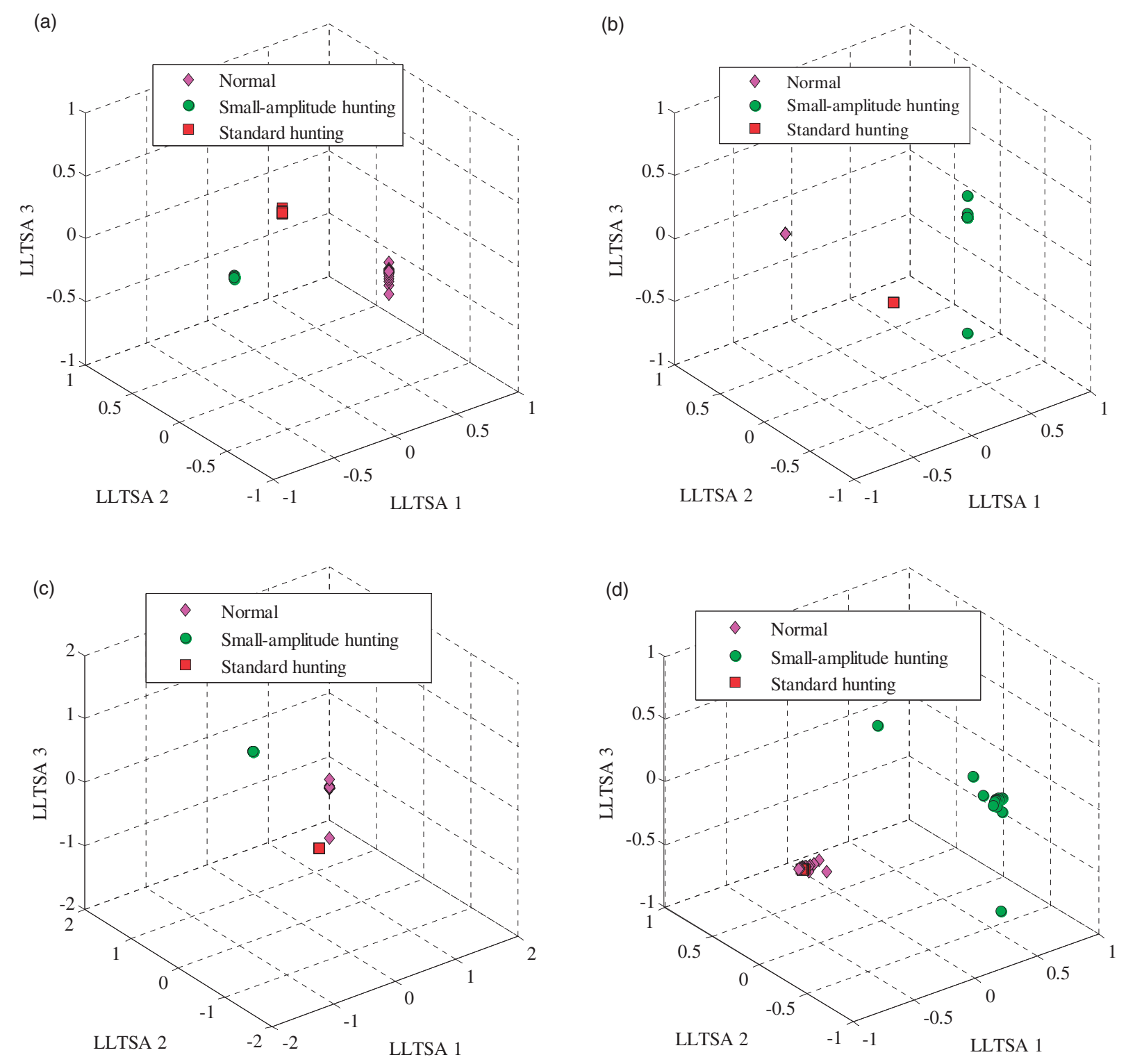

Figure 9. Clustering effect of multi-direction data fusion and IMFR-LLTSA: (a) lateral-longitudinal-vertical, (b) lateral-longitudinal, (c) longitudinal-vertical and (d) lateral-vertical. 
Table 2. Clustering evaluation results and recognition rates of multi-direction data fusion.

\begin{tabular}{|c|c|c|c|c|c|}
\hline \multirow[b]{2}{*}{ Feature extraction method } & \multirow[b]{2}{*}{ Fusion signal } & \multicolumn{3}{|c|}{ Clustering evaluation indices } & \multirow{2}{*}{$\begin{array}{l}\text { Recognition } \\
\text { rate }(\%)\end{array}$} \\
\hline & & $S_{w}$ & $S_{b}$ & $S_{w} / S_{b}$ & \\
\hline \multirow[t]{5}{*}{ IMFR-LLTSA } & Lateral-longitudinal-vertical & 0.2154 & 1.2249 & 0.1758 & 100 \\
\hline & Lateral-longitudinal & 1.9135 & 1.2294 & 1.5579 & 93.33 \\
\hline & Longitudinal-vertical & 1.2126 & 2.0037 & 0.6052 & 66.67 \\
\hline & Lateral-vertical & 8.5703 & 0.2491 & 34.4051 & 66.67 \\
\hline & Lateral & 0.0926 & 0.9836 & $0.094 I$ & 84.44 \\
\hline \multirow[t]{2}{*}{ IMFR-PCA } & Lateral-longitudinal-vertical & 6.7857 & 7.2946 & 0.9300 & 91.11 \\
\hline & Lateral & 0.5625 & 1.2283 & 0.4586 & 80.00 \\
\hline EEMD-energy-LLTSA & Lateral & 3.6831 & 1.1283 & 2.8085 & 71.11 \\
\hline EEMD-SampEn-LLTSA & Lateral & 9.6076 & 0.0982 & 92.3381 & 64.44 \\
\hline
\end{tabular}

data fusion is superior to the methods of the lateral signal, lateral-longitudinal data fusion, lateral-vertical data fusion and the longitudinal-vertical data fusion, and the recognition rate reaches $100 \%$.

Our observations show that when the train speed is at $330-350 \mathrm{~km} / \mathrm{h}$, in the case of convergence, the shortest duration of the small-amplitude hunting is usually 4-6 s; in the case of evolution into supercritical hunting instability, its duration is generally greater than $7 \mathrm{~s}$ (see Figure 2). In the IMFR-LLTSA method, we selected $2 \mathrm{~s}$ as the sampling time of a sample. After training the IMFR-LLTSA-LSSVM network, the average time for identifying the input samples is $1.2246 \mathrm{~s}$. Therefore, the total time is 3.2246 s. Computing facilities: software: MATLAB R2016a; hardware: Intel Core i7-4790K, 4.00 GHz.

For the first convergent small-amplitude hunting, real-time monitoring can also be achieved, but the remaining adjustment time is not long. Fortunately, in practice, this convergent small-amplitude hunting will not cause severe damage to the train. For the second small-amplitude hunting that can evolve into supercritical bifurcation, the $3.2246 \mathrm{~s}$ is much less than $7 \mathrm{~s}$, and the control system has enough time for safety adjustments. The results show that this method can achieve the early warning of the standard hunting.

\section{Conclusion}

In view of the small-amplitude hunting issue, based on the idea of the bogie lateral-longitudinal-vertical data fusion, combining the IMFR-LLTSA feature extraction method, a small-amplitude hunting diagnosis method of high-speed trains is proposed, and the conclusions are drawn as below.

1. When the train runs at high speed, it is prone to small-amplitude hunting. However, the existing monitoring methods rarely involve this motion. In addition, small-amplitude hunting is a 3D motion, and its instability information is shown in the lateral, longitudinal, and vertical vibration signals of the bogie frame. To address these concerns, an idea of bogie frame's lateral-longitudinal-vertical data fusion for small-amplitude hunting diagnosis is proposed.

2. Due to the complex structure and harsh operation environment of trains, massive non-linear signals collected by the monitoring system usually contain multiple nature oscillatory modes, and the single typical feature extraction methods usually cannot characterize these signals. Therefore, an IMFRLLTSA-based feature extracting method is proposed. This method utilizes the self-adaptiveness of EEMD and the excellent dimensionality reduction capability of LLTSA. It has adaptive nonlinear feature extraction capabilities.

3. A small-amplitude hunting diagnosis method combining the idea of the bogie frame's laterallongitudinal-vertical data fusion and IMFRLLTSA is proposed. This method has been validated using the experimental data of the CRH380a running on the Shanghai-Hangzhou railway line. The results show that this method is superior to the single lateral diagnosis method.

Due to the limitation of experimental resources, this paper only studied the CRH380a train. The correct recognition rate of this method under different train models needs to be further verified.

There are two points worthy of further study: (1) Up to now, the small-amplitude hunting issue has not attracted much attention, and a detailed research on its mechanism is yet to be done. In future, the authors would like to explore its mechanism from a dynamics perspective. (2) For the definition of small-amplitude hunting, it is not accurate enough. Hence, the authors would like to use the three-directional vibration signal to define the small-amplitude hunting with clear indicators and attempt to detect small-amplitude hunting through these indicators more efficiently.

\section{Declaration of Conflicting Interests}

The author(s) declared no potential conflicts of interest with respect to the research, authorship, and/or publication of this article. 


\section{Funding}

The author(s) disclosed receipt of the following financial support for the research, authorship, and/or publication of this article: This research work is supported by the National Natural Science Foundation of China (Grant No. 51475387), National Key Technology R\&D Program of China (Grant No. 2009BAG12A01-E03 and 2009BAG12A01-E02), and China Scholarship Council (Grant No. 201707000113). The authors would like to thank China Scholarship Council.

\section{ORCID iD}

Yunguang Ye (D) http://orcid.org/0000-0002-2921-8420

\section{References}

1. Wickens AH. The dynamic stability of railway vehicle wheelsets and bogies having profiled wheels. Int $J$ Solids Struct 1965; 1: 319-341.

2. Wickens AH. Static and dynamic instabilities of bogie railway vehicles with linkage steered wheelsets. Veh Sys Dyn 1996; 26: 1-16.

3. Antali M, Stepan G and Hogan SJ. Kinematic oscillations of railway wheelsets. Multibody Syst Dyn 2015; 34: 259-274.

4. Iwnicki S. Simulation of wheel-rail contact forces. Fatigue Fract Eng Mater Struct 2003; 26: 887-900.

5. Zhang WH, Li Y and Song DL. Design methods for motion stability of high-speed trains. J Southwest Jiaotong Univ 2013; 48: 1-9. (in Chinese).

6. Santamaria J, Vadillo EG and Gomez J. Influence of creep forces on the risk of derailment of railway vehicles. Veh Sys Dyn 2009; 47: 721-752.

7. Iwnicki S. Handbook of railway vehicle dynamics. Boca Raton: CRC Taylor \& Francis, 2006.

8. Köhler G, Scheunemann E and Kolbe T. Die sicherheitstechnische Bedeutung des UIC-Merkblattes 518. Eisenbahn-Revue International 2003; 5: 236-239.

9. Knudsen C, Feldberg R and True H. Bifurcations and chaos in a model of a rolling railway wheelset. Philos Trans A Math Phys Eng Sci 1992; 338: 455-469.

10. Cui DB, Wang HY, Li L, et al. Optimal design of wheel profiles for high-speed trains. Proc IMechE, Part F: J Rail and Rapid Transit 2013; 229: 248-261.

11. Yabuno H, Takano H and Okamoto H. Stabilization control of hunting motion of railway vehicle wheelset using gyroscopic damper. J Vib Control 2008; 14: 209-230.

12. Cooperrider NK. The hunting behavior of conventional railway trucks. J Eng Ind 1972; 94: 752.

13. True $\mathrm{H}$ and Asmund $\mathrm{R}$. The dynamics of a railway freight wagon wheelset with dry friction damping. Veh Sys Dyn 2003; 38: 149-163.

14. Horak D and Wormley DN. Nonlinear stability and tracking of rail passenger trucks. J Dyn Syst Meas Control 1982; 104: 256-263.

15. Bruni S, Vinolas J, Berg M, et al. Modelling of suspension components in a rail vehicle dynamics context. Veh Sys Dyn 2011; 49: 1021-1072.

16. Braghin F, Bruni S and Diana G. Experimental and numerical investigation on the derailment of a railway wheelset with solid axle. Veh Sys Dyn 2006; 44: 305-325.
17. Xu L, Zhai $\mathrm{W}$ and Chen Z. On use of characteristic wavelengths of track irregularities to predict track portions with deteriorated wheel/rail forces. Mech Syst Signal Process 2018; 104: 264-278.

18. Polach O. Characteristic parameters of nonlinear wheel/ rail contact geometry. Veh Sys Dyn 2010; 48: 19-36.

19. Polach O. Application of nonlinear stability analysis in railway vehicle industry. In: Proceeding of the Euromech 500 Colloquium: Non-Smooth Problems in Vehicle System Dynamics, 2010, part 1, vol. 3, pp.15-17. Springer.

20. Polach O. On non-linear methods of bogie stability assessment using computer simulations. Proc IMechE, Part F: J Rail and Rapid Transit 2006; 220: 13-27.

21. D'Souza AF and Tsung WJ. Influence of wheel-rail profiles on the hunting vibrations of rail vehicle trucks. J Vib Acoust Stress Reliab Des 1985; 107: 167.

22. Cai LJ. A real-time monitoring system of high speed train bogie hunting instablilty base on FPGA. Dissertation, Southwest Jiaotong University, China, 2012. (in Chinese).

23. Ye YG, Ning J, Zhong CJ, et al. Forecasting model of hunting instability of high-speed train bogie based on modified ensemble empirical mode decomposition and least squares support vector machine. J China Rail Soc 2018; 40: 38-43. (in Chinese).

24. Ye YG, Ning J, Zhong CJ, et al. Hunting instability of high-speed train diagnosis method based on modified ensemble empirical mode decomposition, Shannon entropy and LSSVM. Appl Res Comp 2017; 34: 1097-1100. (in Chinese).

25. Sun LX. Evaluation method study and theory analysis of lateral running stability and dynamic derailment of highspeed train. Dissertation, Southwest Jiaotong University, China, 2014. (in Chinese).

26. UIC Code 518. Testing and approval of railway vehicles from the point of view of their dynamic behaviour safety - track fatigue - ride quality. 2nd ed. International Union of Railways, Paris, 2003.

27. 2008/232/EC: Commission Decision of 21 February 2008 concerning a technical specification for interoperability relating to the rolling stock sub-system of the trans-European high-speed rail system (notified under document number $\mathrm{C}(2008)$ 648) (Text with EEA relevance). Available at: https://eur-lex.europa.eu/eli/dec/ 2008/232/oj.

28. UIC Kodex 515. Reisezugwagen Laufwerke (1), 2. Ausgabe, 1984.

29. BS EN14363: 2005. Railway applications-testing for the acceptance of running characteristics of railway vehicles-testing of running behaviour and stationary tests. Czech Institute for Normalisation, 2005. Available at: http://store.uni.com/catalogo/index.php/en-143632005.html.

30. TB/T 3188-2007. Technical specification for railway car safety monitor and diagnosis system, China, 2007. (in Chinese). Available at: http://www.csres.com/detail/ 268891.html.

31. Zhai WM, Liu PF, Lin JH, et al. Experimental investigation on vibration behaviour of a $\mathrm{CRH}$ train at speed of $350 \mathrm{~km} / \mathrm{h}$. Int $J$ Rail Transp 2015; 3: 1-16.

32. Ye YG, Ning J, Zhong CJ, et al. Feature analysis of high-speed train bogie hunting instability based on 
modified ensemble empirical mode decomposition and Hilbert transformation. China Meas Test Technol 2016; 42: 120-125. (in Chinese).

33. Ning J, Lin JH and Zhang B. Time-frequency processing of track irregularities in high-speed train. Mech Syst Signal Process 2016; 66-67: 339-348.

34. Wang Y, Xu GH, Liang L, et al. Detection of weak transient signals based on wavelet packet transform and manifold learning for rolling element bearing fault diagnosis. Mech Syst Signal Process 2015; 54-55: 259-276.

35. Bellino A, Fasana A, Garibaldi L, et al. PCA-based detection of damage in time-varying systems. Mech Syst Signal Process 2010; 24: 2250-2260.

36. Tenenbaum JB. A global geometric framework for nonlinear dimensionality reduction. Science 2000; 290: 2319-2323.

37. Roweis ST. Nonlinear dimensionality reduction by locally linear embedding. Science 2000; 290: 2323-2326.

38. Žvokelj M, Zupan S and Prebil I. Multivariate and multiscale monitoring of large-size low-speed bearings using ensemble empirical mode decomposition method combined with principal component analysis. Mech Syst Signal Process 2010; 24: 1049-1067.

39. Ma DY, Wang YJ, Lan CF, et al. Method of assessing the state of a rolling bearing based on the relative compensation distance of multiple-domain features and locally linear embedding. Mech Syst Signal Process 2017; 86: 40-57.

40. Huang YX, Zhang XF, Lee J, et al. Discriminant diffusion maps analysis: A robust manifold learner for dimensionality reduction and its applications in machine condition monitoring and fault diagnosis. Mech Syst Signal Process 2013; 34: 277-297.

41. He QB. Time-frequency manifold for nonlinear feature extraction in machinery fault diagnosis. Mech Syst Signal Process 2013; 35: 200-218.

42. Zhang C, Wang J, Zhao N, et al. Reconstruction and analysis of multi-pose face images based on nonlinear dimensionality reduction. Pattern Recogn 2004; 37: 325-336.

43. Jia F, Lei YG, Lin J, et al. Deep neural networks: a promising tool for fault characteristic mining and intelligent diagnosis of rotating machinery with massive data. Mech Syst Signal Process 2016; 73-74: 303-315.

44. Worden K, Staszewski WJ and Hensman JJ. Natural computing for mechanical systems research: a tutorial overview. Mech Syst Signal Process 2011; 25: 4-111.

45. Jardine AKS, Lin DM and Banjevic D. A review on machinery diagnostics and prognostics implementing condition-based maintenance. Mech Syst Signal Process 2006; 20: 1485-1510.

46. Asr MY, Ettefagh MM, Hassannejad R, et al. Diagnosis of combined faults in rotary machinery by non-naive Bayesian approach. Mech Syst Signal Process 2017; 85: 56-70.

47. $\mathrm{Wu} \mathrm{Z}$ and Huang NE. Ensemble empirical mode decomposition: a noise-assisted data analysis method. Adv Adapt Data Anal 2009; 01: 1-41.

48. Li YZ, Luo DY and Liu SQ. Orthogonal discriminant linear local tangent space alignment for face recognition. Neurocomputing 2009; 72: 1319-1323.

49. Huang NE, Shen Z and Long SR. The Empirical mode decomposition and the Hilbert spectrum for nonlinear and non-stationary time series analysis. Proc R Soc Med 1998; 454: 903-995.

50. Gao Q, Duan C, Fan H, et al. Rotating machine fault diagnosis using empirical mode decomposition. Mech Syst Signal Process 2008; 22: 1072-1081.

51. Fan J, Zhu ZC, Li W, et al. Fault identification of rotor-bearing system based on ensemble empirical mode decomposition and self-zero space projection analysis. J Sound Vib 2014; 333: 3321-3331.

52. Jiang QS, Jia MP, Hu ZJ, et al. Machinery fault diagnosis using supervised manifold learning. Mech Syst Signal Process 2009; 23: 2301-2311.

53. Zhang T, Yang J, Zhao D, et al. Linear local tangent space alignment and application to face recognition. Neurocomputing 2007; 70: 1547-1553.

54. Su ZQ, Tang BP, Liu ZR, et al. Multi-fault diagnosis for rotating machinery based on orthogonal supervised linear local tangent space alignment and least square support vector machine. Neurocomputing 2015; 157: 208-222.

55. Guo YL and Kareem A. System identification through nonstationary data using time-frequency blind source separation. J Sound Vib 2016; 371: 110-131.

\section{Appendix}

\section{Notation}

$\begin{array}{ll}\mathbf{A} & \text { a projection matrix } \\ c_{n}(t) & n \text {th IMF component of } x(t) \text { after EMD } \\ \bar{c}_{n}(t) & n \text {th IMF component of } x(t) \text { after } \\ & \text { EEMD } \\ d & \text { embedding dimension } \\ D & \text { high-dimensional input space } \\ \mathbf{e} & \text { a column vector with all elements are } \\ & \text { equal to 1 } \\ E_{i} & \text { energy of the ith IMF component } \\ E_{s} & \text { total energy of the IMFs } \\ \mathbf{f}_{n} & n \text {th dimension feature vector } \\ h_{1} & \text { difference between } x(t) \text { and } m_{1} \\ \mathbf{H}_{N} & \text { centering matrix } \\ \mathbf{I} & \text { identity matrix } \\ \mathbf{I M F}_{\mathrm{La}, \text { new }} & \text { reconstructed IMFs of } x_{\mathrm{La}}(t) \\ \mathbf{I M F}_{\mathrm{Lo}, \text { new }} & \text { reconstructed IMFs of } x_{\mathrm{Lo}}(t) \\ \mathbf{I M F}_{\mathrm{Ve}, \text { new }} & \text { reconstructed IMFs of } x_{\mathrm{Ve}}(t) \\ k & \text { neighbor number } \\ k_{i} & \text { data length of the IMF function } \\ \mathbf{L}_{i} & \text { a global transformation matrix } \\ m_{+} & \text {upper envelope of } x(t) \\ m_{-} & \text {lower envelope of } x(t) \\ m_{1} & \text { mean of } m_{+} \text {and } m_{-} \\ p & \text { number of the selected samples } \\ q & \text { number of selected IMFs } \\ \mathbf{Q}_{i} & \text { a local transformation matrix } \\ r_{N}(t) & \text { residence of } x(t) \text { after EMD } \\ \bar{r}_{N}(t) & \text { residence of } x(t) \text { after EEMD } \\ S_{b} & \\ & \end{array}$

A

$c_{n}(t)$

$\bar{c}_{n}(t)$

$D$

$E_{i}$

$E_{s}$

$\mathbf{H}_{N}$

$\mathbf{I M F}_{\mathrm{La}, \text { new }}$

$\mathbf{I M F}_{\mathrm{Lo} \text {, new }}$

$\mathbf{I M F}_{\mathrm{Ve}, \text { new }}$

$k$

$k_{i}$

$m$

$m_{-}$

$m_{1}$

$p$

$\mathbf{Q}_{i}$

$r_{N}(t)$

$S_{b}$ a projection matrix

$n$th IMF component of $x(t)$ after

high-dimensional input space

a column vector with all elements are

equal to

total energy of the IMFs

$n$th dimension feature vector

centering matrix

reconstructed IMFs of $x_{\mathrm{La}}(t)$

reconstructed IMFs of $x_{\mathrm{Lo}}(t)$

reconstructed IMFs of $x_{\mathrm{Ve}}(t)$

neighbor number

a global transformation matrix

upper envelope of $x(t)$

lower envelope of $x(t)$

number of the selected samples

number of selected IMFs

a local transformation matrix

between-class scatter 


\begin{tabular}{|c|c|c|c|}
\hline $\begin{array}{l}S_{w} \\
S_{w} / S_{b}\end{array}$ & $\begin{array}{l}\text { within-class scatter } \\
\text { overall clustering effect }\end{array}$ & $y$ & $\begin{array}{l}\text { lateral displacement of the geometric } \\
\text { center of the wheelset }\end{array}$ \\
\hline$x$ & longitudinal displacement of the geo- & $\mathbf{Y}$ & a low-dimensional dataset \\
\hline $\mathbf{x}_{i}$ & $\begin{array}{l}\text { metric center of the wheelset } \\
\text { sample point }\end{array}$ & $z$ & $\begin{array}{l}\text { vertical displacement of the geometric } \\
\text { center of the wheelset }\end{array}$ \\
\hline$x(t)$ & vibration signal in the time domain & & \\
\hline$x_{\mathrm{La}}(t)$ & lateral acceleration of the bogie frame & $\alpha_{i}$ & $\begin{array}{l}\text { ith eigenvector } \\
\text { ith eigenvalue }\end{array}$ \\
\hline$x_{\mathrm{Lo}}(t)$ & longitudinal acceleration of the bogie & $\begin{array}{l}n_{i} \\
\varphi\end{array}$ & roll angle of the wheelset \\
\hline $\begin{array}{l}x_{\mathrm{Ve}}(t) \\
\mathbf{X}\end{array}$ & $\begin{array}{l}\text { frame } \\
\text { vertical acceleration of the bogie frame } \\
\text { a high-dimensional dataset }\end{array}$ & $\psi$ & $\begin{array}{l}\text { yaw angle of the wheelset } \\
\text { pitch angle of the wheelset }\end{array}$ \\
\hline
\end{tabular}

